\section{Competition for laser fusion?}

from a Correspondent

THE incident laser power needed to achieve a significant thermonuclear energy gain in a deuterium-tritium (DT) target which initially has a density near that of the solid state has been variously estimated to lie between $10^{13}$ and $3 \times 10^{15} \mathrm{~W}$; the lower power is appropriate to hollow spherical pellets seeded with materials having a nuclear charge greater than unity, and the higher power to pure, homogeneous spherical DT pellets. The predicted power needed to maximise thermonuclear gain from a given target depends not only on the sophistication and ingenuity of numerical or analytical models, but also on various physical assumptions; these may range from the technological, such as the inclusion of two-dimensional effects induced by small variations in incident light intensity or target symmetry, to the fundamental, such as a self-consistent treatment of non-linear laser-plasma phenomena. (For a good introduction to the subject, the reader is referred to review papers by J. Nuckolls, R. E. Kidder, K. A. Brueckner, R. L. Morse, and others, to be published in Laser Interaction and Related Plasma Phenomena, 3, edit. by $\mathbf{H}$. Schwartz, Plenum Press.)

An interesting example of the interrelation between practical problems and more fundamental effects in laser fusion is afforded by recent radio-frequency and numerical experiments reported by Kim et al. (Phys. Rev. Lett., 33, 886; frequency electric fields within a density cavity, or 'caviton', has been demonstrated. Their experiment simulates the generation of a parametric instability which occurs when a non-uniform laserplasma is driven by a (pump) electric field directed parallel to the density gradient. Although this field component would be zero in an ideally symmetrical 1974 ), in which the trapping of radio-

spherical implosion, it may not be negligible in a practical experiment; the applied field is thus enhanced by linear, resonant conversion to electrostatic modes in regions near the critical density.

A wide range of other laser-plasma effects are also of interest. Consequently predictions of the laser energy needed for significant thermonuclear gain depend on the wavelength $(\lambda)$ of the laser light. For example, Kidder has estimated that, if core-corona decoupling is the limiting physical process in pure DT pellets, laser energy requirements range from $70 \mathrm{~kJ}$ (at $\lambda=0.265 \mu \mathrm{m}$ ) to $3 \mathrm{MJ}$ (at $\lambda=10.6 \mu \mathrm{m}$ ), whilst Nuckolls computes that a $100 \mathrm{~kJ}, 10^{13}$ watt, $\lambda=\frac{1}{2} \mu \mathrm{m}$ laser is required for useful power generation (invited paper given at VIII International Quantum Electronics Conference, San Francisco, June 1974). An economic DT fusion reactor thus requires lasers having energies significantly above those possible at present and with efficiencies of some $15 \%$ (Spalding, Energia Nucleare, 21, 176; 1974), although hybrid fusionfision energy schemes may lower the required laser efficiency (Horoshko, et al., Annals of Nuclear Science and Engineering, in the press). Probably for these reasons, Professor E. Teller recently told an audience at Stanford University that critical experiments in laser fusion may take a generation to complete (New Scientist, 64, 502; 1974).

Competition to lasers as a heat source for target compression in inertial confinement experiments comes from charged-particle beams, which can now be generated with very high electrical efficiencies. Considerable energies have already been delivered to small targets. Typical parameters for some present-day laser and relativistic electron-beam target-compression experiments are compared in Table 1 . Assuming that ablation of a given target is self regulating, with energy deposition governed by the penetrating power of hot coronal electrons, the absorbed power requirement should be

Table 1 Some typical target compression experiments

\begin{tabular}{|c|c|c|c|c|c|}
\hline Beam characteristics & $\begin{array}{l}\text { Number } \\
\text { of beams }\end{array}$ & $\begin{array}{c}\text { Total energy } \\
(J)\end{array}$ & $\begin{array}{l}\text { Incident power } \\
\text { (W) }\end{array}$ & 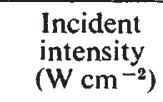 & Ref. $\$$ \\
\hline $\begin{array}{l}1^{*} \lambda=1.06 \mu \mathrm{m} \\
2^{*} \lambda=1.06 \mu \mathrm{m}\end{array}$ & 2 & $\begin{array}{c}230 \\
1,000-3,000\end{array}$ & $2.0 \times 10^{11}$ & $4.0 \times 10^{15}$ & $\begin{array}{l}\text { F1 } \\
\text { F3.2 }\end{array}$ \\
\hline $3^{*} \lambda=1.06 \mu \mathrm{m}$ & 4 & 800 & $2.0 \times 10^{12}$ & $2.0 \times 10^{16}$ & $\mathrm{~F} 4.2$ \\
\hline $4 \lambda=0.53 \mu \mathrm{m}$ & 4 & 140 & - & - & $\mathrm{F} 4.2$ \\
\hline $5^{*} \lambda=1.06 \mu \mathrm{m}$ & 12 & 1,400 & $4.0 \times 10^{11}$ & - & $\mathrm{F} 4.3$ \\
\hline $6^{*} \lambda=1.06 \mu \mathrm{m}$ & 4 & 320 & $1.5 \times 10^{11}$ & $10^{15}$ & $\mathrm{~F} 4.4$ \\
\hline $7^{*} \lambda=1.06 \mu \mathrm{m}$ & 2 & 50 & $1.7 \times 10^{12}$ & - & $\mathrm{F} 4.5$ \\
\hline $8 \dagger \lambda=10.6 \mu \mathrm{m}$ & 1 & 200 & $1.4 \times 10^{11}$ & $7.0 \times 10^{14}$ & $\mathrm{~F} 4.5$ \\
\hline $9 \ddagger \frac{1}{2} \mathrm{MV}$ electrons & 1 & 1,000 & $\sim 5.0 \times 10^{10}$ & $\sim 10^{12}$ & F2.1 \\
\hline $10 \neq 1 \mathrm{MV}$ electrons & 2 & 20,000 & $<10^{12}$ & - & $\mathrm{F} 2.2$ \\
\hline
\end{tabular}

* Nd-glass laser efficiency $<1 \%$

$+\mathrm{CO}_{2}$ laser efficiency currently of order $2 \%$ (can be improved)

$\ddagger$ Electron beam efficiency of order $50 \%$

$\S$ Paper number from the Fifth Conference on Plasma Physics and Controlled Nuclear Fusion Research, IAEA-CN-33, 11-15 November 1974, Tokyo comparable for both laser and particlebeam approaches to inertial confinement. A fundamental problem for the particle approach may thus prove to be the generation of sufficiently high power beams, and their subsequent application at high, uniform intensities to sub-millimetre targets. Winterberg has recently made the interesting suggestion that 1-10 MV heavy ions, having an energy distribution which is instantaneously mono-energetic but which varies with time, might provide a convenient means for axial (time) compression of the beam after passage through a neutralising drift tube (Nature, 251, 44; 1974). Although 300 $\mathrm{keV}$ proton beams of approximately $75 \mathrm{~J}$ energy have recently been produced with an efficiency of $40 \%$ (Bull. Am. phys. Soc., Series II, 19, 871; 1974; papers 2C 14 and 15) no experimental attempt at pulse compression of heavy ion beams has yet been reported. If suitable high-intensity ion-beam techniques could be developed and successfully demonstrated in a compression experiment, the relatively high efficiency with which power could be deposited in the target might provide strong competition to other inertial-confinement approaches.

In summary represent laser techniques seem to offer the most immediate prospect for generating very high density plasmas. Significant laser developments will however be required to permit high-efficiency heating of isolated fuel targets, and to face a potential challenge from charged particle beam techniques for practical fusion energy applications. The likely time scale and scientific challenge to both approaches closely parallels current controlled thermonuclear reactor magnetic confinement programmes.

\section{Probing nuclear band structure}

\author{
from P. E. Hodgson
}

MANY nuclei have low-lying states that are readily interpreted as members of a rotational band. Their energies follow quite closely the values expected from the quantum-mechanical theory of a rigid rotator, and their spins follow typical rotational sequences like $0^{+}, 2^{+}, 4^{+} \ldots$ or $\frac{1}{2}^{+}, 3 / 2^{+}, 5 / 2^{+} \ldots$ depending on the spin of the lowest member of the band. Furthermore, the transition probabilities for the excitation and de-excitation of these bands are found to be in good accord with the same quantum-mechanical theory.

Many such bands have now been found, especially in the regions of the periodic table far from the closed shells where nuclei are frequently deformed. But though it may be easy to identify the lower members of a band it is 